\title{
Relationship between obstructive coronary artery disease and abnormal stress testing in patients with paroxysmal or persistent atrial fibrillation
}

\author{
Gaetano Nucifora $\cdot$ Joanne D. Schuijf $\cdot$ Jacob M. van Werkhoven • \\ Serge A. Trines $\cdot$ Sami Kajander $\cdot$ Laurens F. Tops $\cdot$ Olli Turta $\cdot$ J. Wouter Jukema \\ Joop H. M. Schreur • Mark W. Heijenbrok • Oliver Gaemperli • Philipp A. Kaufmann • \\ Juhani Knuuti $\cdot$ Ernst E. van der Wall $\cdot$ Martin J. Schalij · Jeroen J. Bax
}

Received: 7 July 2010/Accepted: 29 September 2010/Published online: 16 October 2010

(C) The Author(s) 2010. This article is published with open access at Springerlink.com

\begin{abstract}
Atrial fibrillation (AF) has been linked to the presence of underlying coronary artery disease (CAD). However, whether the higher burden of CAD observed in AF patients translates into higher burden of myocardial ischemia is unknown. In 87 patients (71\% male, mean age $61 \pm 10$ years) with paroxysmal or persistent AF and without history of CAD, MSCT coronary angiography and stress testing (exercise ECG test or myocardial perfusion imaging) were performed. CAD was classified as obstructive ( $\geq 50 \%$ luminal narrowing) or not. Stress tests were
\end{abstract}

G. Nucifora - J. D. Schuijf · J. M. van Werkhoven ·

S. A. Trines - L. F. Tops · J. W. Jukema .

E. E. van der Wall · M. J. Schalij · J. J. Bax $(\bowtie)$

Department of Cardiology, Leiden University Medical

Center, Albinusdreef 2, P.O. Box 9600,

2300 RC Leiden, The Netherlands

e-mail: J.J.Bax@lumc.nl

S. Kajander · O. Turta $\cdot$ J. Knuuti

Turku PET Center, Turku University Central Hospital,

Turku, Finland

\section{J. W. Jukema}

The Interuniversity Cardiology Institute of the

Netherlands, Utrecht, the Netherlands

J. H. M. Schreur · M. W. Heijenbrok

Departments of Cardiology and Radiology, Medisch

Centrum Haaglanden, Leidschendam, The Netherlands

O. Gaemperli · P. A. Kaufmann

Cardiovascular Center, University Hospital Zurich,

Zurich, Switzerland classified as normal or abnormal. A population of 122 patients without history of AF, similar to the AF group as to age, gender, symptomatic status and pre-test likelihood, served as a control group. Based on MSCT, $17 \%$ of $\mathrm{AF}$ patients were classified as having no $\mathrm{CAD}$, whereas $43 \%$ showed non-obstructive $\mathrm{CAD}$ and the remaining $40 \%$ had obstructive CAD. A positive stress test was observed in $49 \%$ of $\mathrm{AF}$ patients with obstructive CAD. Among non-AF patients, $34 \%$ were classified as having no CAD, while $41 \%$ showed nonobstructive $\mathrm{CAD}$ and $25 \%$ had obstructive CAD ( $P=0.013$ compared to AF patients). A positive stress test was observed in $48 \%$ of non-AF patients with obstructive CAD. In conclusion, the higher burden of $\mathrm{CAD}$ observed in $\mathrm{AF}$ patients is not associated to higher burden of myocardial ischemia.

Keywords Atrial fibrillation - Coronary artery disease - Multi-slice computed tomography ·

Prevalence $\cdot$ Risk stratification $\cdot$ Stress testing

\section{Introduction}

Atrial fibrillation (AF) has been shown to be an independent risk factor for future coronary artery disease (CAD) events [1-6]. Accordingly, evaluation of AF patients for CAD may potentially be useful, in order to improve their outcome.

Multi-slice computed tomography (MSCT) coronary angiography has emerged as an accurate technique 
for the non-invasive imaging of coronary atherosclerosis [7]. In a recent study using MSCT coronary angiography, a higher prevalence of CAD and obstructive CAD was found among patients with paroxysmal or persistent $\mathrm{AF}$, as compared to patients without a history of AF; in addition, a significant independent relation between $\mathrm{AF}$ and $\mathrm{CAD}$ was observed [8]. However, MSCT coronary angiography does not provide information about the hemodynamic consequences of observed coronary lesions [9, 10]; therefore, whether the higher burden of CAD observed in AF patients is associated also to a higher burden of myocardial ischemia remains to be determined.

Accordingly, the aim of the present study was to evaluate the relation between the evidence of coronary atherosclerosis (by means of MSCT coronary angiography) and the presence of abnormal stress testing among patients with paroxysmal or persistent AF and compare findings to patients without a history of AF.

\section{Methods}

Patient population

A total of 87 patients from the outpatient clinic with a history of paroxysmal $(n=54,62 \%)$ or persistent $(n=33,38 \%) \mathrm{AF}$, referred to MSCT for coronary evaluation, due to an elevated risk profile and/or chest pain, were included. In addition, patients underwent stress testing (exercise ECG testing [EET] or myocardial perfusion imaging [MPI]) within 1 month of MSCT coronary angiography.

The patient population is part of an ongoing study protocol addressing the value of MSCT and other imaging techniques in patients with paroxysmal or persistent AF. From this prospective registry, results addressing the prevalence of CAD by MSCT coronary angiography in AF patients have been recently published [8].

Patients with history of CAD and contraindications to MSCT were excluded. Only patients in sinus rhythm were included, and patients with $\mathrm{AF}$ at the time of SPECT or MPI were excluded. Paroxysmal and persistent AF were diagnosed according to the American Heart Association/American College of Cardiology/European Society of Cardiology criteria [11]. A history of CAD was defined as the presence of previous acute coronary syndrome, percutaneous or surgical coronary revascularization, and/or one or more angiographically documented coronary stenosis $\geq 50 \%$ luminal diameter [9]. Contraindications for MSCT were (1) known allergy to iodinated contrast agent, (2) renal failure, (3) pregnancy.

For each patient, the presence of coronary risk factors (diabetes, systemic hypertension, hypercholesterolemia, positive family history of CAD and cigarette smoking) and symptoms was recorded. In addition, the pre-test likelihood of obstructive CAD was evaluated using the Diamond and Forrester criteria $[12,13]$.

A control group was selected from the clinical database for comparison purposes. Accordingly, 122 patients were included without history of AF and with similar baseline clinical characteristics; these patients were clinically referred to MSCT for coronary evaluation and stress testing within 1 month.

Patients were included at 4 centers in 3 different countries (Leiden University Medical Center, Leiden, the Netherlands; Medisch Centrum Haaglanden, Leidschendam, The Netherlands; Turku PET Center, Turku, Finland; University Hospital Zurich, Zurich, Switzerland).

\section{MSCT data acquisition}

The heart rate and blood pressure were monitored before the examination in each patient. In the absence of contraindications, patients with a heart rate $\geq 65$ beats/min were administered beta-blocking medication (50-100 mg metoprolol, oral or 5-10 mg metoprolol, intravenous).

MSCT coronary angiography was performed with either 16-slice MSCT scanner $(n=39$; Aquilion 16, Toshiba Medical Systems, Japan and Discovery STE, General Electrics, USA) or 64-slice MSCT scanner ( $n=170$; Aquilion 64, Toshiba Medical Systems, Japan, LightSpeed VCT, GE Healthcare, USA and Discovery VCT, General Electrics, USA). The estimated radiation dose was between 10 and $18 \mathrm{mSv}$.

Data were subsequently transferred to dedicated workstations for post-processing and evaluation (Advantage, GE Healthcare, USA; Vitrea 2, Vital Images, USA; and Aquarius, TeraRecon, USA).

\section{MSCT data analysis}

The MSCT data analysis was performed in each center by two experienced observers who had no 
knowledge of the patient's medical history, symptom status and stress testing results; disagreement was solved by consensus or evaluation by a third observer. Standardized MSCT data evaluation methodology and scoring system described below were used in each center.

MSCT coronary angiograms obtained with 16 and 64-slice scanners were evaluated for the presence of obstructive $\mathrm{CAD}$ ( $\geq 50 \%$ luminal narrowing) on a patient and vessel level. For this purpose, both the original axial dataset as well as curved multiplanar reconstructions were used. Each vessel was evaluated for the presence of any atherosclerotic plaque, defined as structures $>1 \mathrm{~mm}^{2}$ within and/or adjacent to the coronary artery lumen, which could be clearly distinguished from the vessel lumen and the surrounding pericardial tissue, as described previously [14]. Subsequently, the vessels were further classified as (1) completely normal, (2) having non-obstructive CAD when atherosclerotic lesions $<50 \%$ of luminal diameter were present or (3) having obstructive CAD when atherosclerotic lesions $\geq 50 \%$ of luminal diameter were present.

The presence of CAD (including obstructive and non-obstructive $\mathrm{CAD}$ ), and obstructive $\mathrm{CAD}$ were evaluated. In addition, the presence of (1) singlevessel disease (obstructive CAD in one vessel); (2) multi-vessel disease (obstructive CAD in more than one vessel) and (3) obstructive CAD in the left main (LM) and/or proximal left anterior descending (LAD) coronary artery was evaluated. Multi-vessel disease and LM and/or proximal LAD disease were considered to represent high-risk features.

\section{Stress testing}

Stress testing was performed in all patients within 1 month of MSCT coronary angiography after an adequate pharmacological wash-out; beta-blockers, long-acting nitrates and calcium channel blockers were discontinued at least $48 \mathrm{~h}$ before the test.

\section{Exercise ECG test}

Symptom-limited EET was performed on a bicycle ergometer according to standard protocols [15]. Patients not able to reach $\geq 85 \%$ age-predicted maximum heart rate in the absence of ischemic changes were not included in the study.
The test was analyzed by an experienced reader who had no knowledge of the MSCT results and was classified as positive or negative for ischemia. The test was considered positive based on the presence of $\geq 0.1 \mathrm{mV}$ horizontal or downsloping ST-segment depression at $80 \mathrm{~ms}$ after the $\mathrm{J}$ point in two contiguous leads during exercise or recovery.

\section{Myocardial perfusion imaging}

Stress-rest MPI was performed with symptom-limited bicycle exercise or pharmacologic (adenosine or dobutamine) stress using either technetium- $99 \mathrm{~m}$ tetrofosmin or technetium-99 m sestamibi. Images were acquired with either a dual-head (Millenium VG \& Hawkeye; GE Healthcare, Waukesha, Wisconsin) or a triple-head (GCA 9300/HG, Toshiba Corp., Japan) single-photon emission computed tomographic (SPECT) camera, and reconstructed into long- and short-axis projections perpendicular to the heart axis. The estimated radiation dose for stress-rest MPI was approximately $7 \mathrm{mSv}$.

The test was analyzed by an experienced reader who had no knowledge of the MSCT results. Perfusion defects were identified on the stress images (segmental tracer activity $<75 \%$ of maximum) and divided into ischemia (reversible defects, with $\geq 10 \%$ increase in tracer uptake on the resting images) or scar tissue (irreversible defects). Accordingly, examinations were classified as being either negative or positive. Positive examinations were further divided into those demonstrating reversible defects and those demonstrating fixed defects. The gated images were used to assess regional wall motion to improve differentiation between perfusion abnormalities and attenuation artifacts [16].

Statistical analysis

Continuous variables are expressed as mean and standard deviation. Categorical variables are expressed as absolute numbers and percentages.

The differences in continuous variables were assessed using the Student $t$ test. Chi-square or Fisher exact test, when appropriate, were computed to test for differences in categorical variables.

A $P$ value $<0.05$ was considered statistically significant. Statistical analyses were performed using SPSS software (version 15.0, SPSS Inc, Chicago, Il, USA). 


\section{Results}

Patient characteristics

Baseline characteristics of each group are shown in Table 1. By definition, AF and non-AF groups did not differ as to mean age $(61 \pm 10$ vs. $59 \pm$ 11 years), male gender ( $71 \%$ vs. $66 \%)$, symptomatic status and pre-test likelihood of CAD. In addition, no difference in coronary risk factor profile was observed between the two groups.

MSCT coronary angiography

Table 2 shows the results of MSCT coronary angiography among AF and non-AF patients. Overall, a significantly higher prevalence of obstructive CAD was observed among AF patients, as compared to non-AF patients $(P=0.013$; Table 2$)$. Single-vessel disease and LM and/or proximal LAD disease were more frequently observed in AF patients $(P=0.027$ and $P=0.003$, respectively; Table 2).

Stress testing

Table 3 shows the stress testing results among AF and non-AF patients.

Symptom-limited EET was performed in 38 (44\%) AF patients and in $48(39 \%)$ non-AF patients. Ischemic ST-segment depression was observed in $15(39 \%) \mathrm{AF}$ patients and in $14(29 \%)$ non-AF patients $(P=0.32$; Table 3). When considering only the symptomatic patients, symptom-limited EET was performed in 13 AF patients and in 23 non-AF patients. In this subgroup of asymptomatic subjects, ischemic ST-segment depression was observed in $8(62 \%)$ AF patients and in $5(22 \%)$ non-AF patients $(P=0.030)$.

Stress-rest MPI was performed in $49(56 \%) \mathrm{AF}$ patients and in $74(61 \%)$ non-AF patients. Symptomlimited bicycle exercise was performed in $21 \mathrm{AF}$
Table 1 Baseline characteristics of the study population
Data are expressed as mean $\pm \mathrm{SD}$ and $n(\%)$

\begin{tabular}{|c|c|c|c|}
\hline & $\begin{array}{l}\text { AF patients } \\
(n=87)\end{array}$ & $\begin{array}{l}\text { Non-AF patients } \\
(n=122)\end{array}$ & $P$ value \\
\hline Age (years) & $61 \pm 10$ & $59 \pm 11$ & 0.25 \\
\hline Male gender & $62(71 \%)$ & $80(66 \%)$ & 0.39 \\
\hline Diabetes & $13(15 \%)$ & $30(25 \%)$ & 0.089 \\
\hline Hypertension & $56(64 \%)$ & $79(65 \%)$ & 0.95 \\
\hline Hypercholesterolemia & $44(51 \%)$ & $53(43 \%)$ & 0.31 \\
\hline Family history of coronary artery disease & $30(35 \%)$ & $50(41 \%)$ & 0.34 \\
\hline Current or previous smoking & $23(26 \%)$ & $39(32 \%)$ & 0.39 \\
\hline$\geq 3$ Coronary risk factors & $26(30 \%)$ & $40(33 \%)$ & 0.66 \\
\hline Body mass index $\left(\mathrm{kg} / \mathrm{m}^{2}\right)$ & $26.4 \pm 3.6$ & $26.4 \pm 3.7$ & 0.97 \\
\hline \multicolumn{4}{|l|}{ Symptoms } \\
\hline Asymptomatic & $45(52 \%)$ & $68(56 \%)$ & \multirow[t]{3}{*}{0.84} \\
\hline Atypical angina & $24(28 \%)$ & $30(25 \%)$ & \\
\hline Typical angina & $18(21 \%)$ & $24(20 \%)$ & \\
\hline \multicolumn{4}{|l|}{ Pre-test likelihood of coronary artery disease } \\
\hline Low & $46(53 \%)$ & $69(57 \%)$ & \multirow[t]{3}{*}{0.63} \\
\hline Intermediate & $23(26 \%)$ & $34(28 \%)$ & \\
\hline High & $18(21 \%)$ & $19(16 \%)$ & \\
\hline \multicolumn{4}{|l|}{ Medical therapy } \\
\hline Digoxin & $8(9 \%)$ & $0(0 \%)$ & 0.001 \\
\hline Beta-blockers & $28(32 \%)$ & $32(26 \%)$ & 0.35 \\
\hline Nitrates & $13(15 \%)$ & $13(11 \%)$ & 0.36 \\
\hline Non-dihydropyridinic calcium antagonists & $9(10 \%)$ & $5(4 \%)$ & 0.075 \\
\hline
\end{tabular}


Table 2 MSCT coronary angiography results in the study population
Data are expressed as mean $\pm \mathrm{SD}$ and $n(\%)$

Table 3 Stress testing results in the study population
Data are expressed as mean $\pm \mathrm{SD}$ and $n(\%)$

\begin{tabular}{lllc}
\hline & $\begin{array}{l}\text { AF patients } \\
(n=87)\end{array}$ & $\begin{array}{l}\text { Non-AF patients } \\
(n=122)\end{array}$ & $P$ value \\
\hline Type of MSCT scanner & $18(21 \%)$ & $21(17 \%)$ & 0.53 \\
$\quad$ 16-slice & $69(79 \%)$ & $101(83 \%)$ & 0.13 \\
64-slice & $64 \pm 7$ & $66 \pm 10$ & \\
$\quad$ Mean heart rate during the scan (beats/min) & & & \\
Prevalence of CAD & $15(17 \%)$ & $41(34 \%)$ & 0.013 \\
$\quad$ Normal coronary arteries & $37(43 \%)$ & $50(41 \%)$ & \\
$\quad$ Non-obstructive CAD & $35(40 \%)$ & $31(25 \%)$ & 0.027 \\
$\quad$ Obstructive CAD & $19(22 \%)$ & $13(11 \%)$ & 0.48 \\
Obstructive single-vessel disease & $16(18 \%)$ & $18(15 \%)$ & 0.003 \\
Multi-vessel disease & $22(25 \%)$ & $12(10 \%)$ & 0.072 \\
LM and/or proximal LAD & $24(28 \%)$ & $21(17 \%)$ & \\
High-risk features & & & \\
\hline
\end{tabular}

\begin{tabular}{|c|c|c|c|}
\hline & $\begin{array}{l}\text { AF patients } \\
(n=87)\end{array}$ & $\begin{array}{l}\text { Non-AF patients } \\
(n=122)\end{array}$ & $P$ value \\
\hline \multicolumn{4}{|l|}{ Type of stress test } \\
\hline Exercise ECG test & $38(44 \%)$ & $48(39 \%)$ & 0.53 \\
\hline Myocardial perfusion imaging & $49(56 \%)$ & $74(61 \%)$ & \\
\hline \multicolumn{4}{|l|}{ Exercise ECG test } \\
\hline Mean peak double product & $28996 \pm 7346$ & $28854 \pm 6678$ & 0.93 \\
\hline Mean peak workload (Watt) & $191 \pm 52$ & $186 \pm 41$ & 0.60 \\
\hline Ischemic ST-segment depression & $15(39 \%)$ & $14(29 \%)$ & 0.32 \\
\hline \multicolumn{4}{|l|}{ Myocardial perfusion imaging } \\
\hline Myocardial perfusion findings & & & 0.47 \\
\hline Normal perfusion & $30(61 \%)$ & $50(68 \%)$ & \\
\hline Reversible perfusion defect & $17(35 \%)$ & $17(23 \%)$ & \\
\hline Fixed perfusion defect & $2(4 \%)$ & $5(7 \%)$ & \\
\hline Reversible and fixed perfusion defects & - & $2(2 \%)$ & \\
\hline \multicolumn{4}{|l|}{ Overall } \\
\hline Negative test & $53(61 \%)$ & $84(69 \%)$ & 0.23 \\
\hline Positive test & $34(39 \%)$ & $38(31 \%)$ & \\
\hline
\end{tabular}

patients and in 11 non-AF patients; in all these patients, $\geq 85 \%$ of maximum age-predicted heart rate was achieved if no stress-induced symptoms or changes in electrocardiogram or blood pressure occurred. Pharmacologic stress using adenosine or dobutamine was applied in 28 AF patients and in 63 non-AF patients.

Thirty $(61 \%)$ AF patients had normal perfusion at both stress and rest. In the remaining 19 (39\%) AF patients, reversible and fixed defects were observed in 17 and 2 patients, respectively. None of the AF patients showed both reversible and fixed defects (Table 3). The prevalence of abnormal MPI scans among non-AF patients was similar. Normal myocardial perfusion was observed in $50(68 \%)$ non-AF patients. In the remaining 24 (32\%) non-AF patients, reversible, fixed and both reversible and fixed defects were observed in 17, 5 and 2 patients, respectively (see Table 3 ). When considering only the symptomatic patients, stress-rest MPI was performed in 29 
symptomatic AF patients and in 31 symptomatic nonAF patients. The prevalence of abnormal MPI scans between these two groups of patients was similar $(8,28 \%$ vs. $11,36 \% ; P=0.58)$.

Overall, considering the combined EET and stressrest MPI results, no statistically significant difference in the prevalence of abnormal stress tests was observed between AF and non-AF patients (39\% vs. $31 \%, P=0.23$; Table 3). Similarly, no statistically significant difference in the prevalence of abnormal stress tests was observed between symptomatic AF and non-AF patients ( $38 \%$ vs. $30 \%, P=0.38$ ).

Relationship between obstructive coronary artery disease and abnormal stress testing

Figure 1 illustrates the relationship between observations on MSCT coronary angiography and stress test results among $\mathrm{AF}$ and non-AF patients. The majority of $\mathrm{AF}$ and non-AF patients with normal coronary arteries had a normal stress test $(87 \%$ vs. $88 \%$; $P=0.90)$. In patients with (any) CAD, 32 (44\%) AF
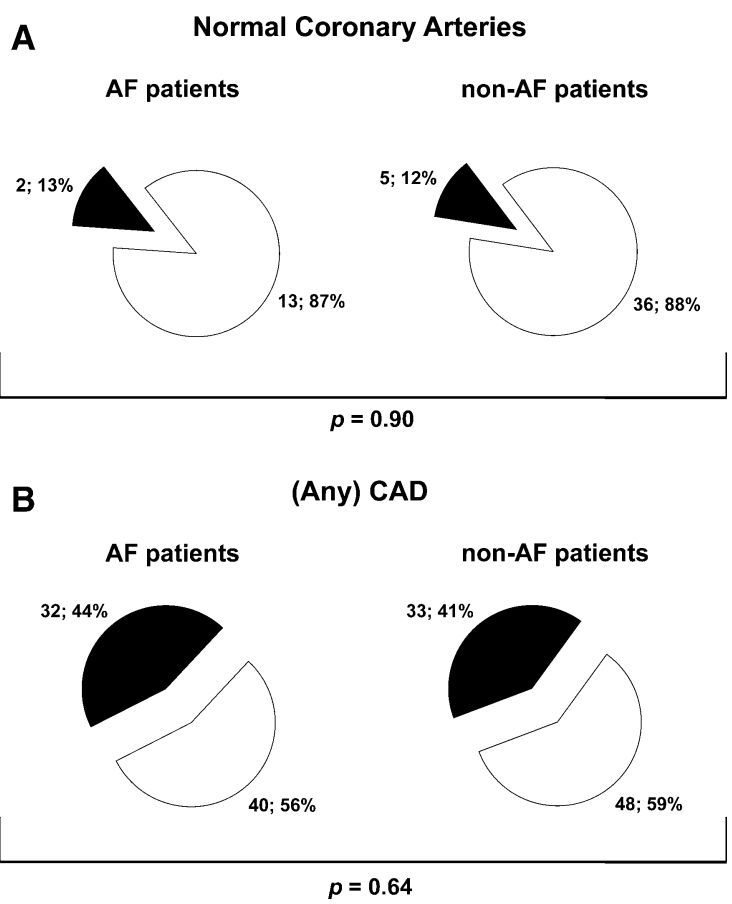

Fig. 1 Pie charts illustrating the relationship between normal coronary arteries (panel a) and any coronary artery disease $(C A D)$ (panel b) identified by MSCT coronary angiography and stress test results among atrial fibrillation $(A F)$ and non- $A F$ patients. White: negative stress test. Black: positive stress test
A

Non-obstructive CAD

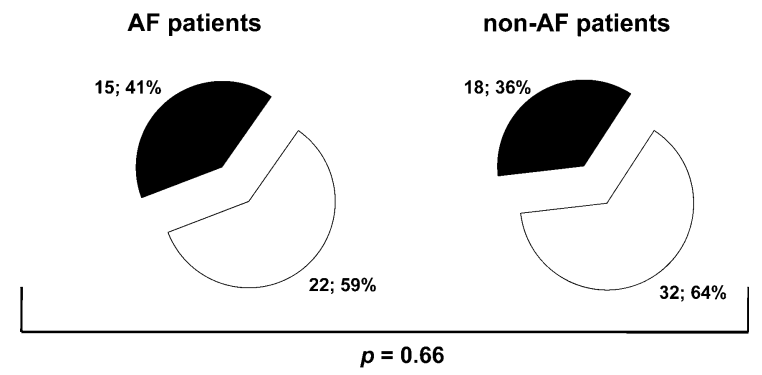

B

Obstructive CAD

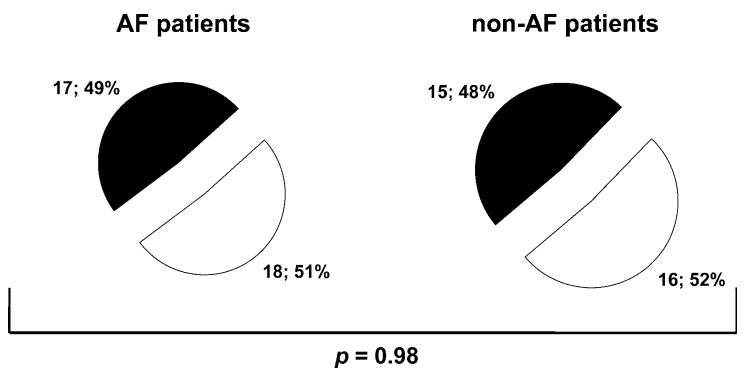

Fig. 2 Pie charts illustrating the relationship between nonobstructive coronary artery disease $(C A D)$ (panel a) and obstructive $C A D$ (panel b) identified by MSCT coronary angiography and stress test results among atrial fibrillation $(A F)$ and non- $A F$ patients. White: negative stress test. Black: positive stress test

patients and $33(41 \%)$ non-AF patients had an abnormal stress test $(P=0.64)$.

Figure 2 illustrates the relationship between nonobstructive and obstructive CAD identified by MSCT coronary angiography and stress test results among $\mathrm{AF}$ and non-AF patients. The majority of AF and non-AF patients with non-obstructive CAD had a normal stress test $(59 \%$ vs. $64 \% ; P=0.66)$. In patients with obstructive CAD, 17 (49\%) AF patients and $15(48 \%)$ non-AF patients had an abnormal stress test $(P=0.98)$.

\section{Discussion}

The results of the present study show that AF patients have a higher prevalence of $\mathrm{CAD}$, and in particular of obstructive $\mathrm{CAD}$, as compared to non-AF patients. However, no difference in the prevalence of abnormal stress testing and of functionally relevant coronary lesions was observed between the two groups. 
Clinical relevance of CAD in AF patients

Previous studies have shown that AF patients have a low risk of $\mathrm{CAD}$ events at the time of first $\mathrm{AF}$ $[17,18]$, but a higher long-term risk, as compared to patients without AF [3, 6]. Aronow et al. [3], for instance, in a prospective study of 1359 patients, demonstrated that $\mathrm{AF}$ patients have a 2.2 times increased probability of developing CAD events during a follow-up of $42 \pm 26$ months, as compared to non-AF patients. A more recent community-based longitudinal cohort study of 2768 patients showed that $\mathrm{AF}$ patients without known CAD represent a high-risk group with increased risk for subsequent new coronary ischemic events and mortality during a follow-up of $6.0 \pm 5.2$ years [6].

These observations raise the question whether routine evaluation of underlying CAD in AF patients should be recommended [19]. Thus far, only few studies addressed this issue. Abidov et al. [20], for instance, assessed the prevalence of CAD in $384 \mathrm{AF}$ patients using stress-rest MPI; a higher prevalence of abnormal MPI studies was observed in AF patients as compared to patients without AF. However, in that study, a non-negligible proportion of enrolled patients had symptoms and/or known CAD, and the observed difference in MPI studies results was mainly related to a higher amount of fixed defects in the AF group. Conversely, Askew et al. [19] showed a similar prevalence of abnormal stress-rest MPI studies in 374 asymptomatic AF patients with no history of CAD, as compared to 374 age- and gender-matched controls without AF. More recently, the prevalence of CAD in 150 AF patients was assessed using MSCT coronary angiography; a significantly higher prevalence of CAD and obstructive CAD was observed among AF patients, as compared to 148 patients with similar age, gender, and pre-test likelihood of obstructive CAD. In addition, AF was independently related to the presence of CAD and obstructive CAD, strengthening the hypothesis that AF could be a marker of advanced coronary atherosclerosis [8].

Atherosclerosis versus abnormal stress testing in $\mathrm{AF}$ patients

Stress testing and MSCT coronary angiography provide different, complementary information about CAD (i.e. evidence of myocardial ischemia and evidence of coronary atherosclerosis, respectively) [9, 10]. However, how these data relate each other in AF patients is still unknown. In the present study, the relation between evidence of coronary atherosclerosis, assessed by means of MSCT coronary angiography, and the presence of abnormal stress testing was assessed among 87 patients with paroxysmal or persistent AF. Findings were compared to 122 patients without a history of AF. In line with the study of Askew et al. [19], a similar prevalence of abnormal stress tests was observed between AF and non-AF patients (39\% vs. $31 \%$ ). In addition, and importantly, no difference in the prevalence of functionally-relevant obstructive coronary lesions was observed between the two groups of patients ( $49 \%$ vs. $48 \%$ ). These data suggest that the higher atherosclerotic burden associated to the presence of AF is not associated with a higher burden of myocardial ischemia.

\section{Clinical implications}

The results of the present and of previous studies suggest that a history of AF, per se, should not represent an indication to stress testing as indiscriminate first-line approach to rule out the presence of CAD [19]. AF patients have indeed a similar prevalence of functionally-relevant coronary lesions as compared to non-AF patients. Nevertheless, a higher burden of subclinical coronary atherosclerosis is observed in patients with $\mathrm{AF}$ and this higher prevalence may potentially explain the previously observed higher long-term risk of CAD event in this group [3, 6]. Accordingly, more aggressive medical therapy and risk factor modification may be justified in AF patients. Further follow-up studies, with follow-up data, are however needed in order to confirm this hypothesis.

Study limitations

This study has several limitations that should be acknowledged. First, it is a case-control study, the limitations of which are well known. Moreover, the patient population is relatively small, including both symptomatic and asymptomatic subjects, and no prognostic data are available; a larger study, with follow-up data, may provide more conclusive information. Second, only patients with paroxysmal or persistent AF were enrolled, while patients with permanent AF were not included. Sixty-four-slice 
MSCT suffers indeed from limited diagnostic accuracy in case of irregular heart rate. More recent generations of dual source MSCT or 320-row scanners could potentially allow imaging of the coronary arteries also in patients with permanent AF [21]. Third, MSCT scanners from different generations as well as manufacturers were used and the stress testing protocol was not standardized, including either symptom-limited EET or stress-rest MPI; this, however, reflects the daily clinical practice, and allows wider applicability to the present observations. In addition, no off-site reading of MSCT coronary angiography and stress testing was performed, possibly influencing inter-observer variability.

\section{Conclusions}

$\mathrm{AF}$ patients have a higher prevalence of $\mathrm{CAD}$, and in particular of obstructive CAD, as compared to non$\mathrm{AF}$ patients. However, the higher burden of CAD observed in AF patients is not associated with a higher burden of myocardial ischemia, as compared to non-AF patients.

Acknowledgments Gaetano Nucifora is financially supported by the Research Fellowship of the European Association of Percutaneous Cardiovascular Interventions (Sophia Antipolis, France). Jacob M. van Werkhoven is financially supported by the Netherlands Society of Cardiology (Utrecht, the Netherlands).

Conflict of interest Martin J. Schalij has research grants from Biotronik (Berlin, Germany), Boston Scientific (Natick, Massachusetts) and Medtronic (Minneapolis, Minnesota). Jeroen J. Bax has research grants from Biotronik (Berlin, Germany), BMS Medical Imaging (North Billerica, Massachusetts), Boston Scientific (Natick, Massachusetts), Edwards Lifesciences (Irvine, California), GE Healthcare (Buckinghamshire, United Kingdom), Medtronic (Minneapolis, Minnesota) and St. Jude Medical (St. Paul, Minnesota). The other authors have nothing to disclose.

Open Access This article is distributed under the terms of the Creative Commons Attribution Noncommercial License which permits any noncommercial use, distribution, and reproduction in any medium, provided the original author(s) and source are credited.

\section{References}

1. Kannel WB, Abbott RD, Savage DD, McNamara PM (1983) Coronary heart disease and atrial fibrillation: the Framingham Study. Am Heart J 106:389-396

2. Krahn AD, Manfreda J, Tate RB, Mathewson FA, Cuddy TE (1995) The natural history of atrial fibrillation: incidence, risk factors, and prognosis in the Manitoba Follow-Up Study. Am J Med 98:476-484

3. Aronow WS, Ahn C, Mercando AD, Epstein S (1995) Correlation of atrial fibrillation, paroxysmal supraventricular tachycardia, and sinus rhythm with incidences of new coronary events in 1,359 patients, mean age 81 years, with heart disease. Am J Cardiol 75:182-184

4. Psaty BM, Manolio TA, Kuller LH, Kronmal RA, Cushman M, Fried LP, White R et al (1997) Incidence of and risk factors for atrial fibrillation in older adults. Circulation 96:2455-2461

5. Kopecky SL, Gersh BJ, McGoon MD, Chu CP, Ilstrup DM, Chesebro JH, Whisnant JP (1999) Lone atrial fibrillation in elderly persons: a marker for cardiovascular risk. Arch Intern Med 159:1118-1122

6. Miyasaka Y, Barnes ME, Gersh BJ, Cha SS, Bailey KR, Seward JB, Iwasaka T et al (2007) Coronary ischemic events after first atrial fibrillation: risk and survival. Am J Med 120:357-363

7. Cademartiri F, La Grutta L, Palumbo A, Malagutti P, Pugliese F, Meijboom WB, Baks T et al (2007) Noninvasive visualization of coronary atherosclerosis: state-ofart. J Cardiovasc Med (Hagerstown) 8:129-137

8. Nucifora G, Schuijf JD, Tops LF, van Werkhoven JM, Kajander S, Jukema JW, Schreur JH et al (2009) Prevalence of coronary artery disease assessed by multislice computed tomography coronary angiography in patients with paroxysmal or persistent atrial fibrillation. Circ Cardiovasc Imaging 2:100-106

9. Schuijf JD, Wijns W, Jukema JW, Atsma DE, de Roos A, Lamb HJ, Stokkel MP et al (2006) Relationship between noninvasive coronary angiography with multi-slice computed tomography and myocardial perfusion imaging. J Am Coll Cardiol 48:2508-2514

10. Petretta M, Costanzo P, Acampa W, Imbriaco M, Ferro A, Filardi PP, Cuocolo A (2008) Noninvasive assessment of coronary anatomy and myocardial perfusion: going toward an integrated imaging approach. J Cardiovasc Med (Hagerstown) 9:977-986

11. Fuster V, Ryden LE, Cannom DS, Crijns HJ, Curtis AB, Ellenbogen KA, Halperin JL et al (2006) ACC/AHA/ESC 2006 guidelines for the management of patients with atrial fibrillation: a report of the American College of Cardiology/American Heart Association Task Force on Practice Guidelines and the European Society of Cardiology Committee for Practice Guidelines (Writing Committee to Revise the 2001 Guidelines for the Management of Patients With Atrial Fibrillation): developed in collaboration with the European Heart Rhythm Association and the Heart Rhythm Society. Circulation 114:e257e354

12. Diamond GA, Forrester JS (1979) Analysis of probability as an aid in the clinical diagnosis of coronary-artery disease. N Engl J Med 300:1350-1358

13. Gibbons RJ, Balady GJ, Bricker JT, Chaitman BR, Fletcher GF, Froelicher VF, Mark DB et al (2002) ACC/AHA 2002 guideline update for exercise testing: summary article: a report of the American College of Cardiology/American Heart Association Task Force on Practice Guidelines (Committee to Update the 1997 Exercise Testing Guidelines). Circulation 106:1883-1892 
14. Leber AW, Knez A, Becker A, Becker C, von Ziegler F, Nikolaou K, Rist C et al (2004) Accuracy of multidetector spiral computed tomography in identifying and differentiating the composition of coronary atherosclerotic plaques: a comparative study with intracoronary ultrasound. J Am Coll Cardiol 43:1241-1247

15. Fletcher GF, Balady G, Froelicher VF, Hartley LH, Haskell WL, Pollock ML (1995) Exercise standards. a statement for healthcare professionals from the american heart association. writing group. Circulation 91:580-615

16. Smanio PE, Watson DD, Segalla DL, Vinson EL, Smith WH, Beller GA (1997) Value of gating of technetium$99 \mathrm{~m}$ sestamibi single-photon emission computed tomographic imaging. J Am Coll Cardiol 30:1687-1692

17. Friedman HZ, Weber-Bornstein N, Deboe SF, Mancini GB (1987) Cardiac care unit admission criteria for suspected acute myocardial infarction in new-onset atrial fibrillation. Am J Cardiol 59:866-869

18. Zimetbaum PJ, Josephson ME, McDonald MJ, McClennen S, Korley V, Ho KK, Papageorgiou P et al (2000)
Incidence and predictors of myocardial infarction among patients with atrial fibrillation. J Am Coll Cardiol 36: 1223-1227

19. Askew JW, Miller TD, Hodge DO, Gibbons RJ (2007) The value of myocardial perfusion single-photon emission computed tomography in screening asymptomatic patients with atrial fibrillation for coronary artery disease. J Am Coll Cardiol 50:1080-1085

20. Abidov A, Hachamovitch R, Rozanski A, Hayes SW, Santos MM, Sciammarella MG, Cohen I et al (2004) Prognostic implications of atrial fibrillation in patients undergoing myocardial perfusion single-photon emission computed tomography. J Am Coll Cardiol 44:1062-1070

21. Marwan M, Pflederer T, Schepis T, Lang A, Muschiol G, Ropers D, Daniel WG, et al. (2010) Accuracy of dualsource computed tomography to identify significant coronary artery disease in patients with atrial fibrillation: comparison with coronary angiography. Eur Heart J. [Epub ahead of print] 